\title{
Síntesis de Aditivos para Biodiesel a partir de Modificaciones Químicas de la Glicerina
}

\author{
Sandra Y. Giraldo, Luis A. Ríos, Alexander Franco y Fernando Cardeño \\ Universidad de Antioquia, Grupo Procesos Fisicoquímicos Aplicados, Sede de Investigaciones \\ Universitarias (SIU), calle 62 No. 52-49, Medellín-Colombia (e-mail: sayagire@yahoo.com)
}

\section{Resumen}

Se muestran los resultados experimentales de la síntesis de cetales de glicerina para su uso como aditivos que mejoren las propiedades de flujo a baja temperatura de un biodiesel de aceite de palma. Se evalúo su producción empleando catalizadores heterogéneos ácidos del tipo resinas de poliestireno sulfonadas (Amberlyst 15, Dowex 50X2) y homogéneos (ácido sulfúrico y ptoluensulfónico). Se analizó el efecto del cetal en las propiedades de flujo a baja temperatura al mezclarse en diferentes proporciones con el biodiesel, evaluando el punto de nube y el punto de fluidez a cada mezcla. Mediante calorimetría diferencial de barrido se evaluó los puntos de cristalización de las mezclas del aditivo con el biodiesel y se comparó con el correspondiente para el biodiesel puro. Se encontró que con el empleo de catalizadores heterogéneos se pueden obtener conversiones por encima del 90\% y la adición de los cetales al biodiesel puede lograr disminuciones de hasta $6^{\circ} \mathrm{C}$ en el punto de nube y fluidez. También, los termogramas de enfriamiento mostraron una disminución efectiva en las temperaturas de cristalización.

\section{Synthesis of Additives for Biodiesel through Chemical Modifications of Glycerol}

\begin{abstract}
In this work the results of the synthesis of glycerin ketals for application as additives to improve the cold flow properties of palm oil biodiesel employing heterogeneous and homogeneous catalyst are shown. Sulfonated polystyrene resins (Amberlyst 15, Dowex 50X2) were employed as heterogeneous catalysts, and homogenous catalysts (sulfuric acid and p-toluensulfonic acid). The effect of amount of ketals on cold flow properties of biodiesel was analyzed through determination of cloud point and pour point for each blend. Through differential scanning calorimetry crystallization points of additive-biodiesel blends were evaluated and compared with those of unmodified biodiesel. It was found that with the use of heterogeneous catalysts, conversions of glycerin above $90 \%$ were obtained. Also, the addition of ketals to biodiesel allows reductions up to $6^{\circ} \mathrm{C}$ in the cloud point and pour point. Also, cooling thermograms showed effective reduction in crystallization temperatures.
\end{abstract}

Keywords: glycerol, ketals, biodiesel, chemical modifications, heterogeneous catalysis 


\section{INTRODUCCIÓN}

El biodiesel ha ganado interés como combustible alternativo debido a la disminución en las fuentes de petróleo y al impacto negativo que este último ha tenido sobre el medio ambiente. Es un combustible renovable, biodegradable y de combustión limpia. En el caso particular de Colombia, las perspectivas para la producción de biodiesel están centradas en el Aceite de Palma, del cual es el 5to productor y sexto exportador mundial (Fedepalma, 2007). El biodiesel es una mezcla de alquilésteres de ácidos grasos, producidos por la transesterificación de triglicéridos con alcoholes de cadena corta, por lo general, en presencia de catalizadores inorgánicos ácidos o básicos homogéneos. La glicerina es el principal subproducto, cuya producción es equivalente a aproximadamente el 10\% en peso del total de biodiesel producido (Bajpai y Tyagi, 2006; Schuchardt et al., 1998). El biodiesel en general comparado con el diesel de petróleo cumple con la mayoría de propiedades fisicoquímicas de este combustible, sin embargo, en lo que se refiere a las propiedades de flujo a baja temperatura (PFBT), presenta valores inferiores, lo que constituye el principal problema para su uso en regiones y en épocas donde la temperatura del ambiente es baja (Knothe et al., 2005; Schumacher et al., 1999). Esto se debe principalmente al tipo de materia prima que se emplee, así, el biodiesel derivado de aceites vegetales compuesto por ácidos grasos saturados presentan los más altos puntos de nube y fluidez (Dunn et al., 1996). El biodiesel de aceite de palma contiene $51.51 \%$ de metilésteres de ácidos grasos saturados mostrando las más deficientes PFBT en comparación con el biodiesel derivado de otros aceites (Fangrui y Milford, 1999; Mihara et al., 2006; Ali et al., 1995). La tabla 1 muestra las propiedades de de nube y fluidez para diferentes alquilesteres.

Tabla 1: PBFT de varios alquilésteres de aceites vegetales (Knothe et al., 2005)

\begin{tabular}{|l|c|c|c|}
\hline Aceite/Alcohol & $\begin{array}{c}\text { Viscosidad } \\
(\mathrm{cP})\end{array}$ & $\begin{array}{c}\text { Punto de nube } \\
\text { PN }\left({ }^{\circ} \mathrm{C}\right)\end{array}$ & $\begin{array}{c}\text { Punto de fluidez } \\
\text { PF }\left({ }^{\circ} \mathrm{C}\right)\end{array}$ \\
\hline Canola/ Metílico & 4.23 & 1.0 & -9.0 \\
\hline Canola/ Etílico & -- & -1.0 & -6.0 \\
\hline Soya/ Metílico & 4.01 & 0.0 & -2.0 \\
\hline Soya/ Etílico & 4.41 & 1.0 & -4.0 \\
\hline Soya/ Butílico & -- & -3.0 & -7.0 \\
\hline Soya/ Isopropílico & -- & -9.0 & -12.0 \\
\hline Girasol/ Metílico & 4.30 & 2.0 & -3.0 \\
\hline Girasol/ Etílico & -- & -1.0 & -5.0 \\
\hline Colza/ Metílico & 3.85 & -2.0 & -9.0 \\
\hline Colza/ Etílico & -- & -2.0 & -15.0 \\
\hline Palma/ Metílico & 4.415 & 16.0 & 12 \\
\hline Palma/ Etílico & -- & 8.0 & 6.0 \\
\hline
\end{tabular}

Se han reportado diferentes estrategias para mejorar las PFBT dentro de las que se destacan: (i) mezcla en bajas proporciones con diesel (Knothe et al., 2005; Rushang y Michael, 2007; Dunn y Bagby, 1995); (ii) empleo de aditivos derivados de alcoholes ramificados o de mayor longitud de cadena (Lee et al., 1995; Rodríguez et al., 2006; Moser y Erhan, 2008); (iii) empleo de la winterización (Dunn et al., 1996; Lee et al., 1996; González et al., 2002); (iv) empleo de aditivos formulados para mejorar las PFBT de combustibles (Schumacher et al., 1999; Bashkatova, 2001); (v) mezcla de biodiesel de diferentes aceites vegetales (Park et al., 2007) y (vi) empleo de aditivos del tipo acetales, cetales y acetatos (Delgado, 2004; Filley, 2004; Delgado, 2008; Bradin, 2007). Los acetales y cetales son compuestos útiles para los combustibles, en tanto que mejoran sus propiedades de combustión ya que son compuestos oxigenados, lo que disminuye las emisiones y mejora las PFBT (Filley, 2004; Noureddini, 2000). Se obtienen por reacción entre alcoholes con aldehídos y cetonas, mediante catálisis homogénea ácida (Meyer et al., 2004; Showler y Darley, 1966), aunque se han reportado estudios con catalizadores heterogéneos ácidos (Deutsch et al., 2007; Augusti et al., 2006; Thomas, 2005).

En este trabajo se reporta la síntesis de cetales cíclicos a partir de la glicerina y su empleo como aditivos para mejorar las PFBT del biodiesel de palma. La síntesis se llevó a cabo evaluando tanto catalizadores heterogéneos como homogéneos. En el caso heterogéneo se emplearon resinas comerciales de intercambio iónico que presentan diferente porcentaje de entrecruzante (Dowex, 
Amberlyst-15) y acidez tipo Brönsted, la tabla 2 muestra algunas características fisicoquímicas de estas resinas . En el caso homogéneo, se emplearon ácido sulfúrico y ácido p-toluensulfónico. A partir de los productos de síntesis, se realizaron mezclas en diferentes porcentajes y se evaluó su efecto en las PFBT del biodiesel de palma, y se determinaron los puntos de cristalización mediante DSC.

Tabla 2: Características de las resinas ácidas empleadas (Skogley y Dobermann, 1996)

\begin{tabular}{|l|l|l|l|l|l|l|l|}
\hline Resina & Descripción & $\begin{array}{l}\text { Comp. } \\
\text { química }\end{array}$ & Acidéz & $\begin{array}{l}\text { Cantidad } \\
\text { Ácida } \\
(\mathrm{meq} / \mathrm{g})\end{array}$ & $\begin{array}{l}\text { BET } \\
(\mathrm{m} 2 / \mathrm{g})\end{array}$ & $\begin{array}{l}\text { Porosidad } \\
\text { mic=micropor } \\
\text { mes=mesopor } \\
\text { mac=macropor }\end{array}$ & $\begin{array}{l}\text { Diám. } \\
\text { prom. } \\
\text { Poro } \\
(\AA)\end{array}$ \\
\hline $\begin{array}{l}\text { Amberlyst } \\
15\end{array}$ & $\begin{array}{l}\text { Macro } \\
\text { reticular }\end{array}$ & $\begin{array}{l}\text { Copolímero } \\
\text { estireno } \\
+20 \% \text { DVB }\end{array}$ & Brönsted & 4,72 & 51 & mes + mac \\
\hline $\begin{array}{l}\text { Dowex } \\
\text { W50X2 }\end{array}$ & $\begin{array}{l}\text { Micro } \\
\text { reticular }\end{array}$ & $\begin{array}{l}\text { Copolímero } \\
\text { estireno } \\
+2 \% \text { DVB }\end{array}$ & Brönsted & 4,3 & $\begin{array}{l}\text { No } \\
\text { aplica }\end{array}$ & mes + mac \\
800
\end{tabular}

\section{PROCEDIMIENTO EXPERIMENTAL}

Los materiales empleados en el presente estudio fueron: Glicerina (99.2\%, Chemi), acetona (98\%, Merck), acido p-toluensulfónico (98\%, Panreac), $\mathrm{H}_{2} \mathrm{SO}_{4}(97 \%$, Merck), resinas ácidas comerciales Dowex W50X2, Amberlyst-15 (Sigma Aldrich) y aceite crudo de palma (empresa extractora de la región).

Biodiesel de aceite de palma

El biodiesel se obtuvo por reacción de transesterificación entre el aceite crudo de palma previamente acondicionado y metanol en una relación molar alcohol: aceite de 12:1, usando $0.7 \%$ de $\mathrm{KOH}$ como catalizador (p/p aceite). La reacción se llevó a cabo en reactores de vidrio de $500 \mathrm{ml}$, provistos con agitación mecánica y sistema de reflujo a una temperatura de $60^{\circ} \mathrm{C}$ durante 1 hora (Benjumea et al., 2004). Posteriormente el biodiesel se lavó y purificó para eliminar las trazas de catalizador, metanol, ácidos grasos libres, jabón y glicerina. Mediante cromatografía gaseosa y normas ASTM se caracterizo el producto obtenido, como se muestra en la tabla 3.

Tabla 3: Caracterización del biodiesel de aceite de palma

\begin{tabular}{|c|c|c|c|c|c|}
\hline \multicolumn{6}{|c|}{ Metiléster de ácidos grasos (\%) } \\
\hline Laúrico & Mirístico & Palmítico & Esteárico & Oleico & Linoleico \\
\hline 0.267 & 1.434 & 46.129 & 3.684 & 37.466 & 11.020 \\
\hline \multicolumn{2}{|c|}{$\begin{array}{c}\text { Densidad } \\
\left(\mathrm{Kg} / \mathrm{m}^{3}\right) A S T M- \\
\text { D4052 }\end{array}$} & $\begin{array}{c}\text { Viscosidad a } 40 \\
{ }^{\circ} \mathrm{C} \text { (cst) ASTM - } \\
\text { D445 }\end{array}$ & $\begin{array}{c}\text { Poder calorífico } \\
\text { (Btu/lb) } \\
\text { ASTM-D240 }\end{array}$ & $\begin{array}{c}\text { Punto de } \\
\text { inflamación }\left({ }^{\circ} \mathrm{C}\right) \\
\text { ASTM-D93 }\end{array}$ & $\begin{array}{l}\text { Numero de } \\
\text { cetano } \\
\text { ASTM-D976 }\end{array}$ \\
\hline \multicolumn{2}{|c|}{864,42} & 4,604 & 17181 & 158 & 54,72 \\
\hline
\end{tabular}

Cetales de glicerina

La síntesis de los cetales se llevo a cabo a escala de laboratorio en reactores de vidrio de $50 \mathrm{ml}$, provistos con sistema de reflujo, control de temperatura y agitación magnética. Se evaluó la conversión de la glicerina y la selectividad hacia el cetal, para los diferentes catalizadores heterogéneos, variando la relación molar acetona:glicerina (3:1 y 6:1), la cantidad de catalizador (1, 3 y $5 \%$ p/p respecto a la glicerina) y el tiempo de reacción (2,4 y 8 h). En el caso de los catalizadores homogéneos la cantidad evaluada fue 0.5 y $1 \%$. Finalmente, se compararon los mejores catalizadores de cada tipo. En la figura 1 se muestra la reacción de la síntesis de cetales. 


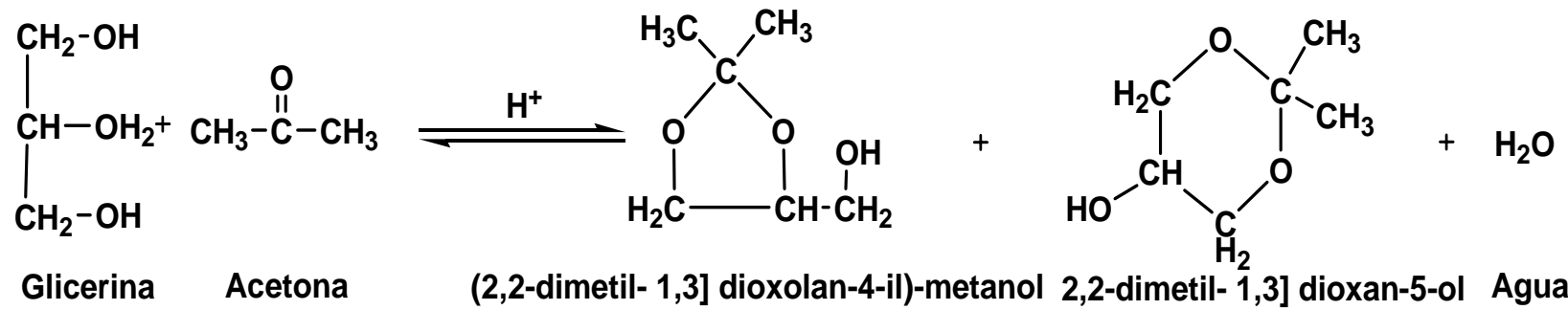

Fig. 1: Reacción de síntesis de cetales de glicerina

\section{Análisis de productos}

Para cuantificar los productos y determinar la conversión de la glicerina, se tomaron muestras a diferentes tiempos de reacción y se inyectaron a un cromatógrafo gaseoso Agilent 6590 provisto con detector TCD y una columna capilar de 60 m AT-WAX, previa neutralización y/o filtración del catalizador. Para la cuantificación se construyeron curvas de calibración empleando estándares de los reactivos y de los productos.

Mezclas del aditivo con el biodiesel de palma y PFBT

Se realizaron mezclas del dioxolano con el biodiesel de palma en proporciones de 1-10\% en volumen. Las PFBT consideradas en este estudio fueron el PN (temperatura a la cual se observa una nube de cristales) y el PF (temperatura a la cual el líquido deja de fluir cuando se pone en un plano horizontal). Dichas propiedades se determinaron siguiendo procedimientos descritos en las normas ASTM D2500 y D97.

\section{Análisis por calorimetría diferencial de barrido (DSC)}

Se determinaron los puntos de cristalización del biodiesel de palma a las diferentes muestras variando el aditivo mediante un equipo TA Instruments. Las muestras se calentaron a $50^{\circ} \mathrm{C}$ por 5 min y luego se enfriaron a razón de $5^{\circ} \mathrm{C} / \mathrm{min}$, desde $50^{\circ} \mathrm{C}$ hasta $-70^{\circ} \mathrm{C}$.

\section{RESULTADOS Y DISCUSIÓN}

A continuación se muestran los resultados de la síntesis de los cetales mediante la reacción de la glicerina con la acetona empleando catalizadores homogéneos y heterogéneos y la posterior evaluación de los productos en mezclas con el biodiesel de aceite de palma como aditivos para mejorar el punto de nube y de fluidez del biodiesel así como los resultados de DSC para las diferentes mezclas.

\section{Reacción con catálisis heterogénea}

A continuación las figuras 2-5 muestran la conversión de glicerina vs tiempo para las resinas Dowex y Amberlyst, para cada variable evaluada.

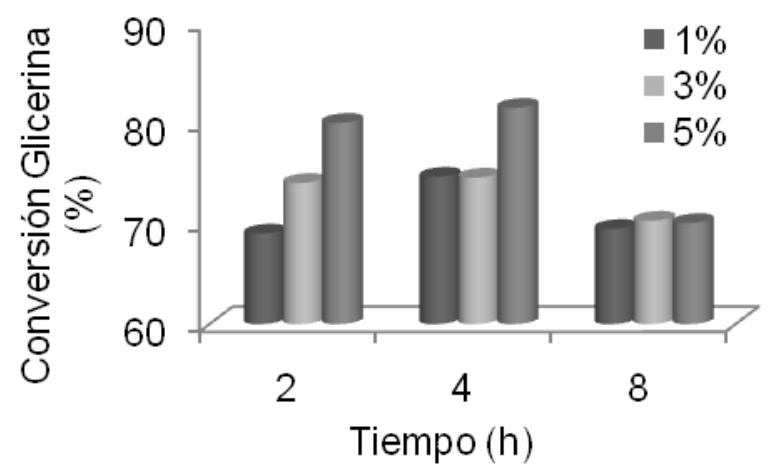

Fig 2: Conversión empleando Dowex. Relación molar 3:1

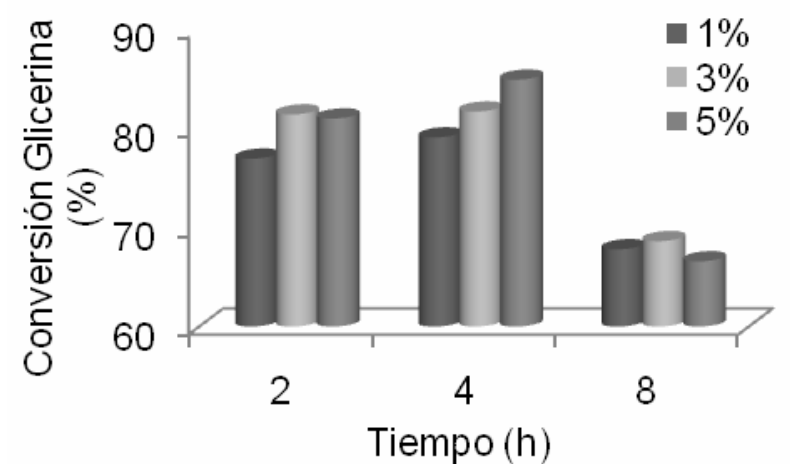

Fig 3: Conversión empleando Dowex. Relación molar 6:1

Como se observa en las figuras 2-5, la conversión de la glicerina, aumenta con el aumento en la cantidad de resina y la relación molar. Con respecto al tiempo de reacción, la conversión se hace máxima en 4 horas y presenta un decaimiento en 8 horas de reacción, lo cual puede deberse a que 
el proceso de síntesis se efectúa en un sistema de reflujo, en el cual el agua formada en el proceso retorna al sistema de reacción lo que favorece la hidrólisis de los productos. Otra razón puede estar asociada a la disminución en la actividad de las resinas por la inclusión de agua en los poros, lo que genera el desplazamiento de la acetona y disminuye la reacción, finalmente debido a la pérdida de la por difusión en la fase gaseosa respecto al tiempo.

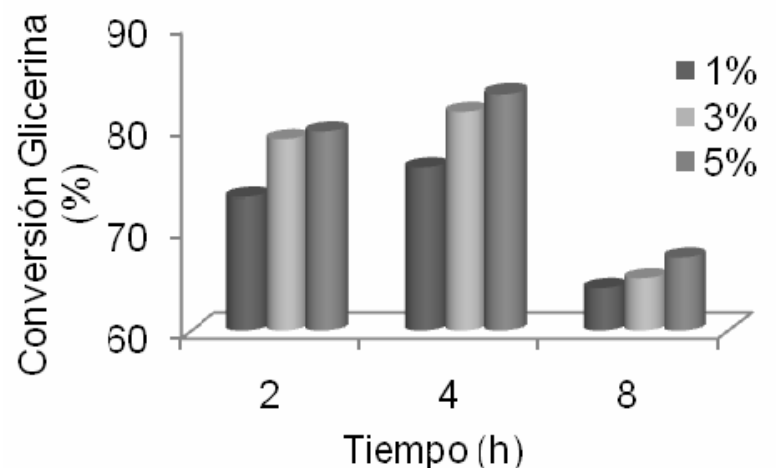

Fig 4: Conversión empleando Amberlyst-15. Relación molar 3:1

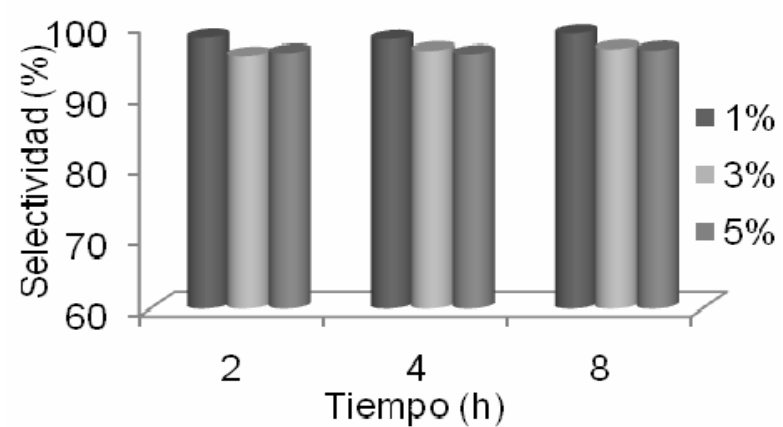

Fig 6: Selectividad cetal 5. Catalizador Amberlyst15

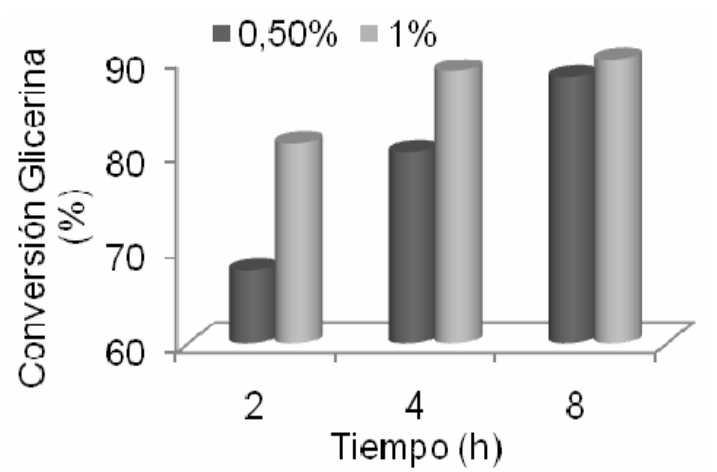

Fig 8: Conversión con $\mathrm{H}_{2} \mathrm{SO}_{4}$. Relación molar 3:1

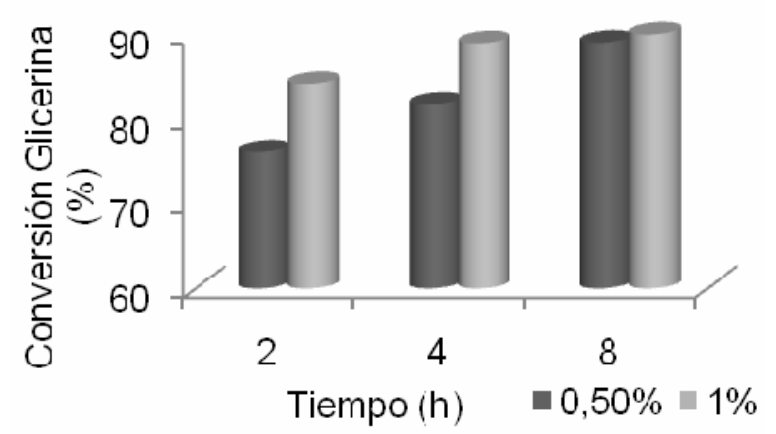

Fig 10: Conversión con A. p-toluensulfónico. Relación molar 3:1

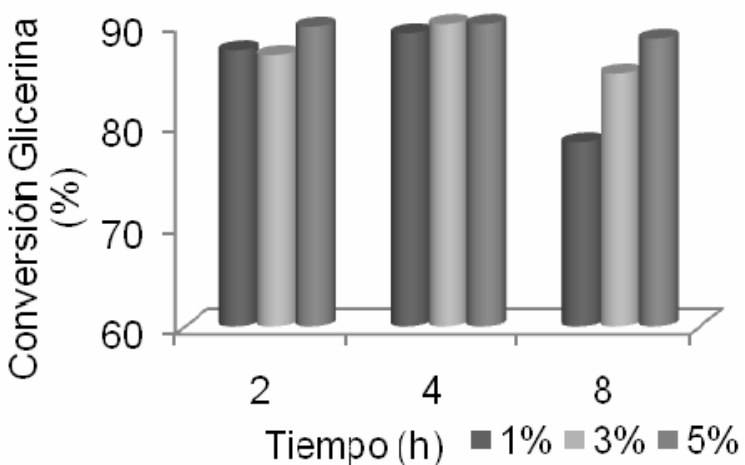

Fig 5: Conversión empleando Amberlyst-15. Relación molar 6:1

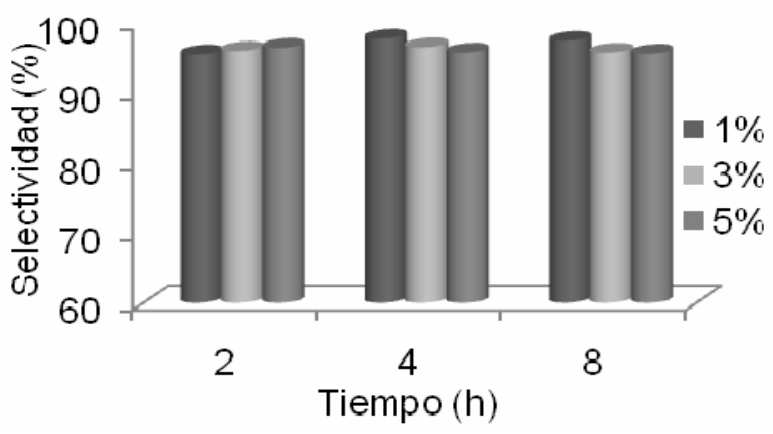

Fig 7: Selectividad cetal 5. Catalizador Dowex

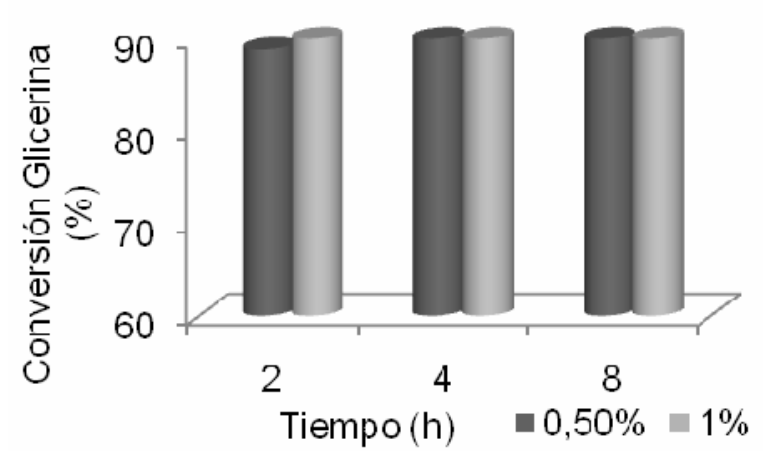

Fig 9: Conversión con $\mathrm{H}_{2} \mathrm{SO}_{4}$. Relación molar 6:1

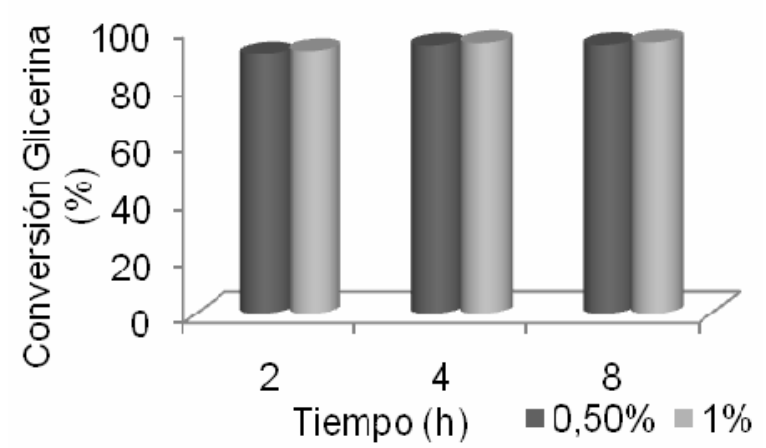

Fig 11: Conversión con A. p-toluensulfónico. Relación molar 6:1 
Comparando los resultados mostrados en las figuras 2-5, se puede observar que la resina Amberlyst muestra la mayor conversión debido principalmente a que los problemas difusionales asociados al nivel de entrecruzamiento, son menos notorios en las resinas del tipo macro reticular, por lo que el contacto íntimo con los reactivos es mayor y la presencia de agua es menos dañina, contrario ocurre en las resinas micro reticulares como la Dowex cuyos sitios activos están en el interior de la resina, y su afinidad por el agua es mayor. Las figuras 6 y 7 muestran los resultados obtenidos para selectividad hacia el compuesto dioxolano o cetal de cinco vértices (cetal 5) de cada una de las resinas empleadas.

En cuanto a la selectividad hacia el cetal cíclico de cinco miembros ((2,2-dimetil-[1,3] dioxolan-4-il)metanol, cetal 5), en las figuras 6 y 7 se observa que no existe una variación, de una resina a otra y la selectividad se mantiene en promedio en $96 \%$, con lo que se puede considerar que se obtiene un producto puro.

\section{Reacción con catálisis homogénea}

En las figuras 8-11, muestran los resultados de conversión vs tiempo para la reacción empleando catálisis homogénea con $\mathrm{H}_{2} \mathrm{SO}_{4}$ y acido p-toluensulfónico. De acuerdo con estas, para los catalizadores homogéneos, la conversión aumenta con el aumento en la relación molar, cantidad de catalizador y tiempo de reacción. Tambien se observa que al aumentar la relación molar el cambio en conversión no es muy significativo con el auemnto en la cantidad de catalizador. Siendo el ácido ptoluensulfónico el catalizador que muestra los mejores resultados, con conversiones de la glicerina por encima del $68 \%$, en poco tiempo de reacción.

Las figuras 12 y 13 muestran que ambos catalizadores homogéneos presentan similar desempeño en cuanto a la selectividad del dioxolano o cetal 5 (entre 96 y $97 \%$ )

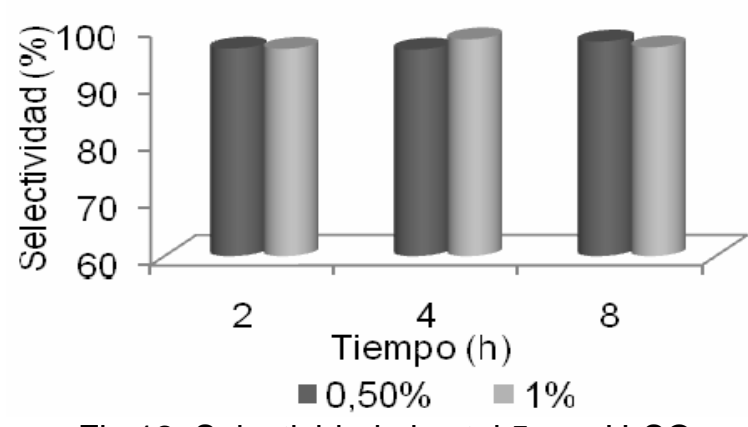

Fig 12: Selectividad al cetal 5 con $\mathrm{H}_{2} \mathrm{SO}_{4}$

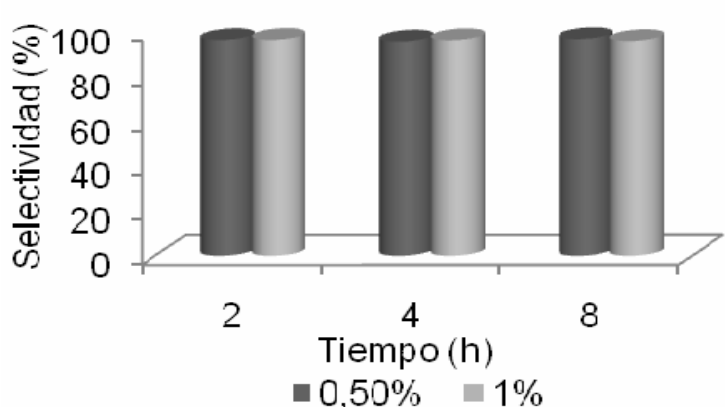

Fig 13: Selectividad al cetal 5 con ácido ptoluensulfónico

Teniendo en cuenta los resultados para la catálisis homogénea y heterogénea, los mejores catalizadores para cada tipo son la resina Amberlyst y el ácido p-toluensulfónico. Se realiza una comparación entre estos dos catalizadores, empleando $1 \%$ de catalizador, variando la relacion molar acetona:glicerina y el tiempo de reaccion, los resultados obtenidos se aprecian en la figura 14:

Como puede observarse, la resina Amberlyst puede sustituir el catalizador homogéneo, ya que la diferencia en cuanto a conversión no es muy significativa, lo que podría ser ventajoso, ya que se harían ahorros en proceso al no ser necesarias etapas de neutralización para extraer el catalizador, además el catalizador heterogéneo puede reutilizarse de nuevo, lo que se traduce en beneficios ambientales.

\section{Calorimetría diferencial de barrido (DSC)}

En general hay dos picos exotérmicos distintivos presentes en los termogramas mostrados en la figura 10. El primer pico exotérmico es el punto de cristalización de los compuestos saturados del biodiesel, y es el de mayor temperatura, el segundo pico a menor temperatura corresponde a la fracción insaturada. El orden descendente de las temperaturas de de cristalización para las mezclas es el siguiente:

Biodiesel>Biodiesel+1\% cetal $5>$ Biodiesel+3\% cetal $5>$ Biodiesel+5\% cetal $5>$ Biodiesel+10\% cetal 5 


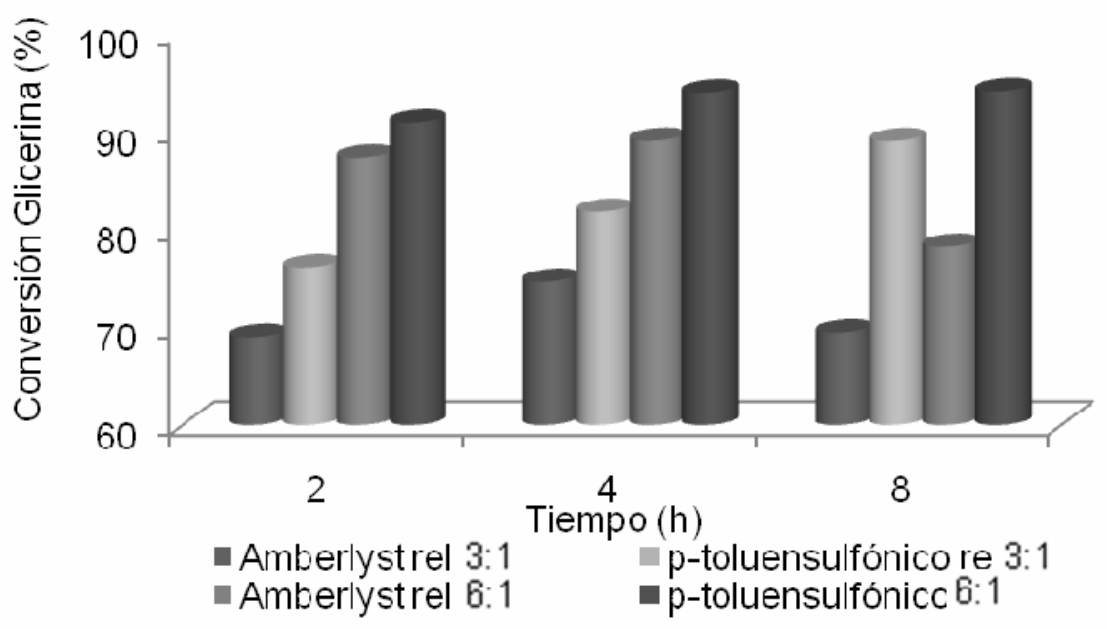

Fig 14: Comparación Amberlyst y p-toluensulfónico

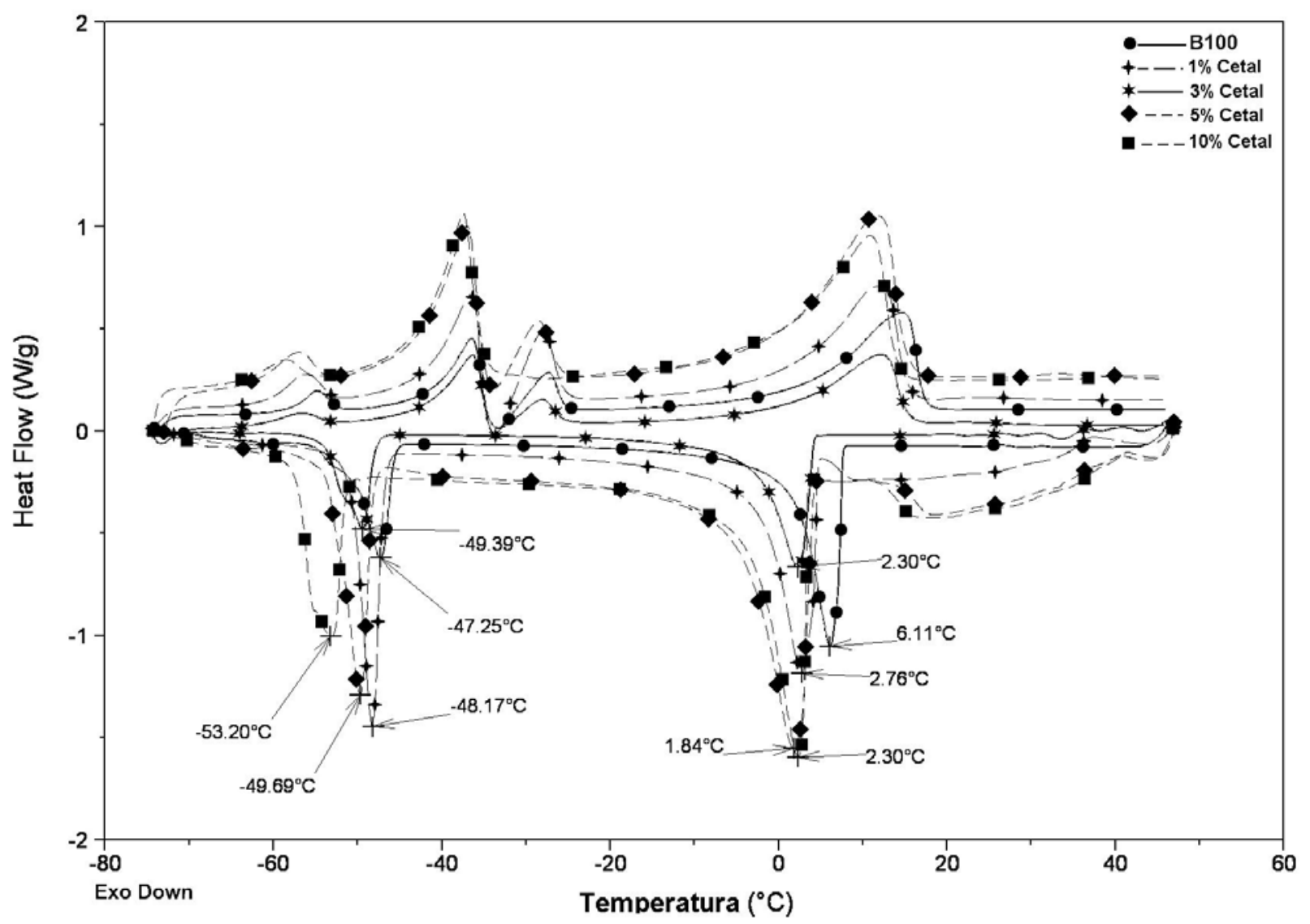

Fig 15: Comparación Biodiesel de palma puro vs biodiesel con cetal 5

Como se observa en la figura 15, el cetal provoca una disminución en la temperatura de cristalización de los compuestos saturados e insaturados. A medida que aumenta la cantidad de cetal en la mezcla, se presenta un desplazamiento de la curva hacia la izquierda.

Propiedades de flujo en frío de las mezclas

En la tabla 4 se muestran las PFBT medidas para la mezcla del biodiesel con el cetal producido, en diferentes proporciones. Se puede observar que el PN y el PF mejoran a medida que aumenta la cantidad del aditivo en la mezcla y son mejores que los respectivos para el biodiesel de aceite de palma puro, esto se explica debido a que el aditivo afecta las formas cristalinas de los compuestos saturados presentes y disminuye la formación de lamelas propias de estos. 
Tabla 4: PFBT para las mezclas con cetal 5

\begin{tabular}{|l|c|c|}
\hline Muestra & $\mathrm{PN}\left({ }^{\circ} \mathrm{C}\right)$ & $\mathrm{PF}\left({ }^{\circ} \mathrm{C}\right)$ \\
\hline B100 & 16 & 14 \\
\hline $1 \%$ cetal & 12 & 11 \\
\hline $3 \%$ cetal & 11 & 10 \\
\hline $5 \%$ cetal & 11 & 9 \\
\hline $10 \%$ cetal & 10 & 8 \\
\hline
\end{tabular}

Puede observarse que las muestras poseen PN y PF que disminuyen al aumentar la cantidad de aditivo. El punto de nube más bajo corresponde a la mezcla con $10 \%$ de acetal. El punto de nube y de fluidez se logra disminuir hasta en $6^{\circ} \mathrm{C}$.

\section{CONCLUSIONES}

Los catalizadores heterogéneos (Amberlyst 15 y Dowex) presentaron buena actividad catalítica para la síntesis de los cetales a partir de la glicerina. Se obtuvieron conversiones superiores a $69 \%$ y selectividad hacia el cetal 5 de 96\%, para ambas resinas. Para estos catalizadores se puede afirmar que un aumento en la hidrofobicidad de la resina aumenta la actividad catalítica, sin embargo, la presencia de agua en el sistema de reflujo, favorece la reacción de hidrólisis, lo que implica, la caída de la conversión en tiempos superiores a 4 horas.

En el caso de las reacciones en presencia de catalizadores homogéneos, el acido p-toluensulfónico mostró los mejores resultados, con valores de conversión superiores al $76 \%$ con respecto al $69 \%$ del acido sulfúrico, lo cual puede explicarse debido a la estructura química de ambos compuestos, el ptoluensulfónico presenta menos afinidad al agua debido a la presencia del grupo aromático que disminuye su polaridad lo que garantiza su mayor disponibilidad en la fase orgánica, a diferencia del acido sulfúrico que prefiere desplazarse a la fase acuosa.

Comparando la resina Amberlyst-15 y el ácido p-toluensulfónico, se puede concluir que ambos presentan conversiones similares, y en el caso del catalizador heterogéneo, hay ventajas adicionales en la purificación ya que implica menores etapas de proceso, además, esta resina puede ser reutilizada.

Los aditivos del tipo cetales derivados de la glicerina mezclado con el biodiesel de palma permitieron mejorar las PFBT, reduciendo el PN y el PF hasta en $6^{\circ} \mathrm{C}$. Un valor adecuado de aditivo suficiente para disminuir estas propiedades es del 3\%, ya que adiciones superiores no tienen un impacto significativo en la reducción del PN y PF. Los resultados de DSC, para la mezcla con aditivos del tipo cetal, modifica la temperatura de cristalización del biocombustible, desplazando a la izquierda los picos exotérmicos de los termogramas con respecto al correspondiente del biodiesel puro, lo que evidencia el desempeño del aditivo.

La fase glicerina de los procesos de obtención de biodiesel pueden ser una fuente alternativa de materia prima para la producción de los cetales, lo que conduciría a mejorar la rentabilidad del proceso de biodiesel ya que se estaría empleando un subproducto con alto volumen de producción, sin embargo, es necesario adecuar esta glicerina.

\section{AGRADECIMIENTOS}

A la Universidad de Antioquia y al grupo Procesos Fisicoquímicos Aplicados de la Facultad de Ingeniería, por el apoyo y por las instalaciones ofrecidas para llevar a cabo este trabajo, a Colciencias y al Ministerio de Agricultura y Desarrollo Rural por su financiación.

\section{REFERENCIAS}

Ali, Y., M. Hanna y S. Cuppett; Fuel Properties of Tallow and soybean oil esters. J Am. Oil Chem. Soc.: 72(12), 1557-1564 (1995). 
Augusti, R. y otros cuatro autores; Atmospheric pressure eberlin transacetilation reactions in the heterogeneous liquid/gas phase. Intern. Journal of Mass Spectrometry: 253 (3), 281-287 (2006).

Bajpai, D. y V.K. Tyagi; Biodiesel: Source, production, composition, properties and its benefits. J. Oleo Sci.: 55 (10), 487-502 (2006).

Bashkatova, S.; Composites depressant additive for diesel fuels. Chemistry and Technology of Fuels and Oils: 37 (3), 177-178 (2001).

Benjumea, P.N; J.R. Agudelo y G.J. Cano; Estudio experimental de las variables que afectan la reacción de transesterificación del aceite crudo de palma para la producción de biodiesel. Scientia et Technica: X, 169-175 (2004).

Bradin, D.; Improved biodiesel fuel, additives and lubbricants. WO 2007027669(2007).

Delgado, J.; Use of glycerine tri-acetate as additive of biodiesel fuel compositions. EP 1331260(2008).

Delgado, J.; Procedimiento para producir combustibles biodiesel con propiedades mejoradas a baja temperatura. ES 2201894A1(2004).

Deutsch, J., A. Martin y $\mathrm{H}$. Lieske; Investigations on heterogeneously catalized condensations of glycerol to cyclic acetals. Journal of Catalysis: 245(2), 428-435 (2007).

Dunn, R. y M. Bagby; Low-Temperature Properties of Triglyceride-Based Diesel Fuels: Transesterified Methyl Esters and Petroleum Middle Distillate/Ester Blends. J. Am. Oil Chem. Soc.: 71(8), 895-904 (1995).

Dunn, R., M. Shockley y M. Bagby; Improving the low temperature properties of alternative diesel fuels: vegetable oil derived methyl esters. J. Am. Oil Chem. Soc.: 73 (12), 1719-1728 (1996).

Fangrui, M. y A. Milford; Biodiesel production: a review. Bioresource Technology: 70 , 1-15 (1999).

Fedepalma, Federación Nacional de Cultivadores de Palma. www.fedepalma.org (2007). Acesso: Agosto de 2007.

Filley, J.; New biolubricants from vegetable oil cyclic acetals of methyl 9,10-dihydroxystearate. Bioresource Technology: 96, 551-555 (2004).

González M. E.R. Howard, J.J. Leahy y B. Rice; Winterization of waste cooking oil methyl ester to improve cold temperature fuel properties. Fuel:. 81(1), 33-39 (2002).

Knothe, G., J. Vangerpen y J. Krahl; The Biodiesel Handbook. Illinois: AOCS Press. (2005).

Lee, I., L. Johnson y E. Hammond; Use of Branched chain esters to reduce the crystallization temperature of biodiesel. J. Am. Oil. Chem. Soc.: 72(10), 1155-1160 (1995).

Lee, L., E. Hammond y L. Johnson; Reducing the crystallization temperature of biodiesel by winterizing methyl soyate. JAOCS: 73 (5), 631-636 (1996).

Meyer, O.; Preparation of ketal. US 01152920-A1(2004).

Mihara, H. y otros cinco autores; Effect of crystallization temperature of palm oil on its crystalization: $\mathrm{J}$. Oleo Sci.: 55 (8), 397-402 (2006).

Moser, B y S. Erhan; Branched chain derivatives of alkyl oleates: Tribological, rheological, oxidation, and low temperature properties. Fuel: 87 (10), 2253-2257 (2008).

Noureddini, H.; Process for producing biodiésel fuel with reduced viscosity and a cloud point below 32 deg. Fahrenheit. US 6.015.440(2000). 
Park, J.; D. Kim y J. Lee; Blending effects of biodiesels on oxidation stability and low temperature flow properties. Bioresource Technology: 99(5), 1196-1203 (2007).

Rodriguez, J. y otros cinco autores; Correlating Chemical Structure and Physical Properties of Vegetable Oil Esters. JAOCS: 83(4), 353-357 (2006).

Rushang, M. y J. Michael; Flow properties of biodiesel fuel blends at low temperatures. Fuel: 86(1), 143-151 (2007).

Schuchardt, U., R. Sercheli y R. Vargas; Transesterification of Vegetable Oils: a Review. J. Braz. Chem. Soc.:, 9 (1), 199-210 (1998).

Schumacher, L., W. Wetherell y J. Fisher; Cold flow properties of biodiesel and its blends with diesel fuel. Annual international ASAE meeting (ASAE, Ed.) Toronto, Canada (1999).

Showler, A. y P. Darley; Condensation products of glycerol with aldehydes and ketones. 2-substituted m-dioxolan-5-ols and 1,3-dioxolane-4-methanols. University of Medical Sciences, Faculty of Medical Science, University of Medical Sciences, Bangkok, 427-440 (1966).

Skogley, E. y A. Dobermann; A. Synthetic Ion-Exchange Resins: Soil and Environmental Studies. Journal or Environmental Quality: 25(1),13-24 (1996).

Thomas, B; P. Sreedharan y S. Sankaran; Synthesis of dimethyl acetal of ketones: design of solid acid catalyst for one-pot acetalization reaction. Microp. and Mesop. Materials: 80(1), 65-72 (2005). 